\title{
5W2H+M: A Broad Gamification Design Process But Focused on Motivation
}

\author{
Gabriel Guebarra Conejo, Isabela Gasparini, Marcelo da Silva Hounsell
}

${ }^{1}$ Department of Computer Science - Graduate Program in Applied Computing Santa Catarina State University

Joinville - SC - Brazil

gabrielgconejo@gmail.com, \{isabela.gasparini,marcelo.hounsell\}@udesc.br

\begin{abstract}
Gamification has been increasingly implemented in systems to motivate users. To assist designers in the gamification process there are several frameworks, some of them address motivation in gamification superficially while others focus on motivation and are not broad enough. This work presents the $5 \mathrm{~W} 2 \mathrm{H}+\mathrm{M}$ framework and its use to redesign an already gamified system. Game elements were implemented to motivate users to increase the frequency and quantity of responses; longevity of use; feeling of accompaniment, and; feeling of belonging on the users. An evaluation was conducted with a specialist and showed that the frequency, amount of questions and feeling of belonging improved. However, the longevity and accompaniment can be further addressed.
\end{abstract}

Resumo. A gamificação é cada vez mais utilizada em sistemas computacionais com o objetivo de motivar os usuários. Para auxiliar o processo da gamificação, existem diversos frameworks, alguns abordam a motivação superficialmente enquanto outros se aprofundam apenas em motivação e não são abrangentes o suficiente. Este artigo apresenta o framework $5 \mathrm{~W} 2 \mathrm{H}+\mathrm{M}$ e sua utilização no redesign de um sistema gamificado. Elementos de jogos foram implementados para motivar o aumento da frequência e quantidade de respostas; longevidade do uso; sensação de acompanhamento e; sentimento de pertencimento. Uma avaliação realizada com um especialista mostrou que a frequência, quantidade de respostas e sentimento de pertencimento melhoraram. Porém, a longevidade e sensação de acompanhamento ainda podem ser abordados.

\section{Introduction}

Gamification is the use of game design elements in contexts that are not ludic [Deterding et al. 2011]. In the perspective of motivation theories, the study of the gamification process shows positive results in the users of computational systems [Brühlmann 2013]. Self-Determination Theory (SDT) is a motivation theory broadly used in gamification processes [Deci and Ryan 2008]. This theory presents motivation as: extrinsic motivation that results from external factors such as prizes; intrinsic motivation that results from internal factors such as satisfaction, and; amotivation which is the lack of motivation. SDT presents basic psychological needs that are necessary to motivate intrinsically and extrinsically: autonomy, which is the need to feel in control of the task; competence, which is the need of progress, and; relatedness, which is the need to connect with other people [Ryan and Deci 2000]. To achieve intrinsic motivation all three of the 
psychological needs must be satisfied and, to achieve extrinsic motivation only competence and relatedness must be satisfied [Ryan and Deci 2000].

To help the gamification design and evaluation processes there are several frameworks that can be used. These frameworks may be focused on some gamification aspects such as motivation or can be broad, comprehending other gamification aspects such as persuasion, fun and engagement, to name a few. On one hand, it was observed that the broader frameworks did not cover motivation in a profound way, and on the other hand, focused frameworks that covered motivation did not cover other aspects of the gamification process.

This paper presents the $5 \mathrm{~W} 2 \mathrm{H}+\mathrm{M}$ framework, which is a broad framework with its motivational aspects extended, and its use to redesign the gamification of a system that was already gamified. Then an evaluation was conducted with a specialist to verify the new gamification effects in the system.

\section{Gamification}

Game elements present the game characteristics that can be applied when gamifying a system [Ryan and Deci 2000]. These game elements are not implemented with the purpose to create a complete game experience and, because of that, cannot be measured the same way as a complete game [Hassenzahl and Tractinsky 2006].

Game elements can be divided in mechanics, dynamics and components, also known as the MDC model [Werbach and Hunter 2012]: Mechanics are basic processes that move the action; Dynamics are aspects not implementable of the gamified system and are abstract elements; Components are specific instances of dynamics and mechanics [Werbach and Hunter 2012]. Dynamics are the more abstract game elements and can be achieved by mechanics that are achieved by components. Some game dynamics are: Rules, emotions, progression and relationships [Werbach and Hunter 2012]. Each dynamic is achieved by one or more mechanic. Mechanics are basic processes less abstract than dynamics and are responsible for the motion of action in the gamified system. Some mechanics are: Chances, competition, challenges, cooperation, competition, feedback, victory states, transactions and rewards [Werbach and Hunter 2012]. Each mechanic can be achieved by one or more game component. Components are specific dynamic and mechanic instances and are directly implemented in the gamified system. Some examples of game components are: Badges, avatars, points, presents, missions, levels, emblems and classification tables [Werbach and Hunter 2012]. Al three describe the user experience in the system [Hassenzahl and Tractinsky 2006].

\section{Self-Determination Theory (SDT)}

SDT is a widely used motivation theory in the gamification processes and has in its core the distinction between autonomous and controlled motivation [Deci and Ryan 2008]: Autonomous motivation comprehends intrinsic motivation and activities in which the individual integrates activities in their self-sense; Controlled motivation comprehends extrinsic motivation and activities that are external to the individual. Intrinsic motivation is executing an activity for its inherent satisfactions rather than some separate consequence and is internally regulated; Extrinsic motivation is promoted by external factors such as prizes and is externally regulated. SDT also presents three basic psychological needs 
which are necessary to achieve intrinsic and extrinsic motivations. Basic psychological needs are defined as [Ryan and Deci 2000, Mekler et al. 2017, Aparicio et al. 2012]:

- Autonomy: In which the causality locus is internal, that is, to satisfy this need the individual must feel that he is doing the task because he chose to do it and not because he was led to do it. It relates to the increase in intrinsic motivation and can be satisfied by game elements such as avatar, user control and, configurable interfaces;

- Competence: it is the known extension of an action to achieve the desired goals in the environment. Some game elements that satisfy competence are: points, levels, classification tables, positive feedback;

- Relatedness: It is the need to interact and be connected to someone. To satisfy this need there must be some interaction of some type with someone and the individual must not be isolated. Some game elements that satisfy the need of relatedness are: cooperation, competition, presents, classification tables, and chat systems.

The theory also argues that to be intrinsically motivated all three needs must be satisfied and to be extrinsically motivated at least competence and relatedness must be satisfied. There is also the concept of purpose that is related to intrinsic motivation. Purpose is doing an action that has meaning to the individual that did it [Pink 2011].

\section{Related Works}

A literature review of the frameworks that can be found and which gamification aspects these frameworks cover was presented by Mora et al. (2015). Only 2 frameworks found in the research were applied to gamify or evaluate the gamification of a system. One of them was the 5W2H framework [Klock 2017] that was used to gamify an online learning system, but motivation was not measured in any way. This framework was created using Mora's review to cover as much gamification aspects as possible. The Octalysis framework was used to categorize mobile applications and did not measure motivation either [Ewais and Alluhaidan 2015]. An evaluation of specific gamification elements was conducted, measuring their effects in intrinsic motivation [Mekler et al. 2017, Hanus and Fox 2015]. Intrinsic motivation was also evaluated in a gamified course and was compared with a non-gamified course [Banfield and Wilkerson 2014]. All of these work show that gamification can be promising to motivate but it is not always the case. Therefore, more investigation on whether it is good or not to use certain game elements in certain contexts is required. Some works did not use any kind of framework to design gamification.

Above mentioned researches show that there is a need of more studies that use a gamification framework as well as an evaluation of motivation after using the framework to see the effects in the user motivation after using a gamified system

\section{The Framework $5 \mathrm{~W} 2 \mathrm{H}$}

5W2H Framework is a user centered, generic, flexible and broad gamification framework that addresses core gamification principles taking advantage of several frameworks that came before it [Klock et al. 2016]. It is composed of seven dimensions:

- "Who?": Has the objective to identify who are the users of the project that compose the target audience. This dimension points out characteristics from the user such as sex, age, culture and objectives; 
- "What?": This dimension points out the behaviors that the target audience should have while using the gamified system. It guides the creation of stimuli to help the realization of this behaviors and tasks;

- "Why?": This dimension is related to the stimuli that will be generated in the users by the interaction with the gamified system. It approaches motivation intrinsically and extrinsically and what is the duration of the stimuli that will generate motivation in users;

- "When?": It identifies what are the appropriate situations in which users will need to be stimulated to the desired behaviors. This dimension presents the players' journey and the stimuli frequency and strength (such as points);

- "How?": This dimension is responsible for helping to choose game elements that will be implemented in the gamified system;

- "Where?": This dimension is where prototypes and the implementation will be made using Human Computer Interaction (HCI) and software engineering to achieve this goal;

- "How Much?": This last dimension is responsible for measuring how much the gamification was able to stimulate the desired behaviors in the target audience.

$5 \mathrm{~W} 2 \mathrm{H}$ was selected as a starting point because it is one of the most complete framework regarding the gamification design, but it lacks details on building a motivational environment.

\section{Framework 5W2H+M}

To deepen the motivational aspects of the $5 \mathrm{~W} 2 \mathrm{H}$ framework towards a more motivational outcome four of the original dimensions of the framework were modified: "Who?", "Why?", "How?" and "How Much?" [Conejo 2018]. Each one of these four dimensions was altered without changing the end goal and use of the framework.

\section{1. "Who?"}

The dimension "Who?" identifies the target audience's characteristics. Initially, there were no motivational characteristics addressed by this dimension. Purpose, Amotivation and Motivation factors were included to help identify possible motivational characteristics in the target audience. Purpose addresses what will be the significance of the gamification in users' routine; amotivation factors identifies possible situations in which the gamification may have a negative impact in users and; motivation factors identifies situations in which the gamification may have a possible impact in users routine.

To help identify these characteristics the use of HCI design techniques such as interviews, questionnaires, focal groups and user observation is advised.

\section{2. "Why?"}

The dimension "Why?" identifies the stimuli that will be generated in the users in order to perform the desired tasks and have the desired behaviors inside the gamified system. This dimension presents three core stimuli that are: engagement, fun and motivation.

The motivation stimulus is presented by its duration (short and long term) and origin (intrinsic or extrinsic). As the SDT suggests, to achieve intrinsic and extrinsic motivations it is necessary to satisfy some basic psychological needs. The satisfaction of the 
needs of autonomy, competence and relatedness were implemented into this dimension to further help the gamification process in the generation of the desired motivation stimulus, be it intrinsic, extrinsic or both. Each psychological need can be satisfied with some game element and the implementation of these needs will help better choose each game element. This dimension is responsible for the UX in the system, that is why it is necessary to use game design and interaction design so that the gamified system may achieve and generate the desired stimuli.

\section{3. "How?"}

This dimension aims to design the gamification so that the stimuli are generated, and the user has the desired behavior inside the gamified system. The dimension is responsible for helping choosing game elements that will be implemented in the system. These game elements are based in the MDC model.

Each basic psychological need can be satisfied by one or more game element. The extension made in this dimension categorizes game elements by each psychological need. This helps selecting right game elements to generate the desired motivation stimuli.

To achieve what the "How?" dimension proposes the participation of specialists such as game designers, software engineers, HCI specialists and systems analysts is necessary so that the project may be viable for implementation.

\section{4. "How Much?”}

The last dimension that was extended was the "How Much?" dimension. This dimension aims to evaluate the gamification. It evaluates how much the gamification was able to stimulate the desired behaviors in given situations presented in the system.

This dimension did not present any metrics to evaluate motivation so, the Intrinsic Motivation Inventory (IMI) is suggested [Ryan et al. 1983]. IMI is composed by several subscales that measure aspects such as perceived choice, perceived competence, relatedness, enjoyment and others. These metrics are used to evaluate how much an individual is intrinsically motivated to do the task and is a well-known tool to evaluate motivation.

Besides the IMI it is possible to use other metrics the designer sees fit such as interviews, other scales, questionnaires, field studies and others. After the evaluation is complete, another iteration of the framework may be applied if the desired results were not achieved to further polish the gamification implemented.

\section{5W2H+M Applied on the ConneCT System}

In order to use the extended framework $5 \mathrm{~W} 2 \mathrm{H}+\mathrm{M}$ in a real situation, the ConneCT accompaniment system was chosen. ConneCT is a gamified system that aims to assist monitoring of drug addicts with questions about the state of their life to know the risk of relapse. Healthcare agents receive daily updates about the addicts that they follow.

ConneCT was gamified by the first version of $5 \mathrm{~W} 2 \mathrm{H}$ to achieve three desired behaviors: high frequency of answers; longevity of use and; high amount of questions answered daily. The gamification consisted of missions, an individual ranking system, points and little feedback. All dimensions of the 5W2H+M framework were applied to redesign and implement ConneCT new version. 
Dimension "Who?" addressed the target audiences' characteristics through an interview with specialists on drug addiction that are healthcare agents was conducted so that information could be gathered. Information about gender, age, purchasing capability, schooling, motivation and amotivation factors were gathered and are as follows: Gender: Most of the of the addicts are male; Age: Between 15 and 50 years old; Purchasing Capability: The majority could afford a smartphone; Schooling: The majority could write and read; Motivation Factor: Be able to report their situation in a less embarrassing way; Amotivation Factor: Threatened anonymity and boredom; Purpose: Use the system as means of opening up about their situation and to keep a diary.

The anonymity is a crucial part of the system because of the context that it was applied and should be respected not only because of being an amotivation factor but because of legal reasons too.

Dimension "What?" is responsible for identifying the desired behaviors that the addicts should have while using the system. The frequency and amount of answers as well as the longevity of the use of the system were already objectives with actions identified, so, the feeling of belonging and the feeling of being accompanied by the healthcare agents were added as objectives. Expected actions to achieve all goals are:

- Frequency: The person must answer the questions every day;

- Amount: Answer all the questions presented in a given day;

- Longevity: Use the system for as long as possible;

- Accompaniment: Note the feedback messages presented while using the system;

- Belonging: Gain points to climb in the team scoreboard.

Dimension "Why?" is responsible for the influence of the gamification on the user. The main stimuli desired is motivation so that users may use the system for a long period of time returning everyday to answer questions.

As SDT states, intrinsic motivation tends to have better results in a long period of time. Knowing that, it was chosen to stimulate all three basic psychological needs of autonomy, competence and relatedness. The first version of ConneCT only had extrinsic stimuli having a great emphasis on game elements that satisfied competence only.

It is expected that with elements that satisfy autonomy and make the user know he/she is not alone using the system, all three basic psychological needs are satisfied and intrinsic motivation would be more likely achieved.

The "When?" dimension identifies the moments in which the desired behavior will be emphasized. As the desired behavior is not complex, requiring only answering questions, the emphasis were designed to achieve important behaviors:

- Answer one question: This behavior will transmit valuable information to the healthcare agent;

- Answer various questions in a given day: This behavior will give extra valuable information to the healthcare agent;

- Answer everyday: To know precisely how is the state of the addict it is necessary a constant flow of information.

To achieve these, answering in consecutive days; answering at least one question a day and; answering all questions for consecutive days will be encouraged by the system. 
The incentives are continuous and fixed, that is, the same type of incentive will be applied continuously in each situation.

On the dimension "How?" all game elements were chosen based on the desired stimuli and emphasis that will be applied. First, the dynamics were chosen based on the desired stimuli, after that the mechanics and finally the components. Table 1 presents each stimuli with its dynamic, mechanic and component chosen.

Table 1. Game Elements implemented On The System, blue components were added to the system, red components already existed but were modified, green components were already in the system and suffered little to none alteration.

\begin{tabular}{|l|l|l|l|}
\hline Stimuli & Dynamics & Mechanics & Components \\
\hline Autonomy & Emotion & Customization & Avatar, Nickname \\
\hline \multirow{3}{*}{ Competence } & \multirow{2}{*}{ Progression } & Rewards & Emblems, Points \\
\cline { 3 - 4 } & & Challenges & Missions, Levels, Points, Emblems \\
\cline { 2 - 4 } & Rules & Feedback & Points, Messages \\
\hline \multirow{2}{*}{ Relatedness } & \multirow{2}{*}{ Relationships } & Competition & Points, Classification Tables \\
\cline { 3 - 4 } & & Cooperation & Teams, Classification Tables \\
\hline
\end{tabular}

Besides the chosen game elements, a leveling system was designed to make easier for the user to see his/her progress: each question answered will yield a sum of points.

On the dimension "Where?", ConneCT was implemented with a soccer fantasy theme because most users are men and as it is used in Brazil, soccer fans. Each game element was implemented with the fantasy in mind. As an example the points are called goals and each level is a new tournament that the player will participate.

Each day the person will have to answer several questions, in the first version of the system it was not possible to choose more questions to answer so, to further increase autonomy the user now can respond more questions besides the required questions of that day, and they can also answer the same questions again.

A profile tab, as seen in Figure 1, was added that allows users to choose an avatar, nickname, team, points and level, and; a ranking of the team, was added instead of only the individual's one. Feedback messages were also implemented to give more information and foster more answers and, messages that pop up when the user does not answer in a given day. All messages use a language that alludes to the fantasy chosen to further immerse the user in the gamification aspect of the fantasy.

On the dimension "How Much?", an evaluation was conducted with a psychologist that is a healthcare agent responsible to accompany drug addicts in their rehabilitation. The specialist had already used the first gamified version of the system with a group of addicts. The evaluation protocol was composed by three use cases: first the specialist used the system freely; then the specialist used the system with a fake user account that supposedly used the system for almost a year; the final use case was with a fake user account that was using the system for the first time. This enabled the specialist to experience all feedback messages and features of the system.

After the test protocol an interview was conducted with questions based on the IMI 


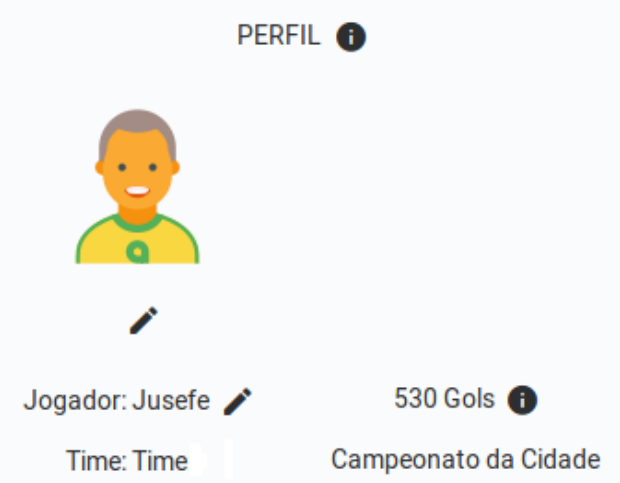

(a) User profile with an avatar, nickname team name, points and level.

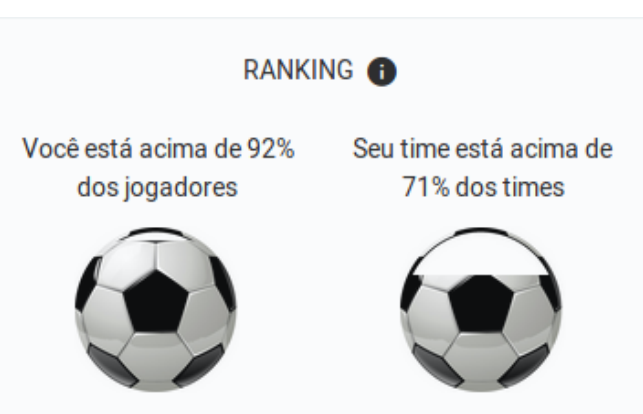

(b) Individual ranking and team ranking.

Figure 1. Two additions made in the ConneCT System.

scale [Ryan et al. 1983]. There were questions about the enjoyment, autonomy, competence, relatedness and tension to evaluate the experience the specialist had with the system and if the system had the desired effects regarding motivation for longevity, frequency and number of answers, feeling of belonging and accompaniment.

The test protocol and the interview with the psychologist revealed that the changes made to the system were noticed and the answers related to autonomy showed that new features such as the possibility to answer the questions again could satisfy autonomy. Regarding competence, the leveling system and feedback were the elements that the specialist though had the most impact an could satisfy this psychological need. And, regarding relatedness, the specialist pointed out that the team ranking and being part of a team were the most important elements added to the system because the feeling of belonging is a crucial part in rehabilitation.

Regarding the objectives of the system, the specialist pointed out that the frequency and amount of questions would be achieved by the possibility to choose to answer again and by feedback messages encouragement; the longevity was unclear and need to be tested in a long period and; the feeling of belonging could be achieved by the team ranking. The specialist did not see the accompaniment sensation being satisfied, possibly because he did not pay attention to the feedback messages (closing them before reading).

\section{Conclusion}

This paper presented an extension of the $5 \mathrm{~W} 2 \mathrm{H}$ framework called $5 \mathrm{~W} 2 \mathrm{H}+\mathrm{M}$. The extended framework helped to choose game elements to implement on the ConneCT system to stimulate the basic psychological needs of autonomy, competence and relatedness and facilitated the gamification project to be implemented when focused on users' motivation. The resulting system was tested with a specialist in psychology that works with addicts' accompaniment. An interview was conducted with the specialist with questions based on the IMI scale and by his answers it is possible to say that the ConneCT system has a good chance to achieve some of its goals. We concluded that the $5 \mathrm{~W} 2 \mathrm{H}+\mathrm{M}$ framework was an effective tool to design the gamification of a system with emphasis on motivational aspects. Future work would be to test the gamified system with other psychologists as 
well as evaluating it from the users point of view.

As shown in the related works, research that extended a gamification framework was not found, only one work presented a framework with its application and only a few works revolved around the application of existing frameworks was found. This work not only extended an already existing framework but applied it in a system.

\section{Acknowledgements}

The authors would like to thank CNPq for the research grant on Technololgy Development and Innovation (DT-CNPq); FAPESC (public call FAPESC/CNPq No. 06/2016 support the infrastructure of CTI for young researchers, project T.O. No.: 2017TR1755 - Ambientes Inteligentes Educacionais com Integração de Técnicas de Learning Analytics e de Gamificação), T.O. No. 2019TR712 and; partial fundind from Coordenação de Aperfeiçoamento de Pessoal de Nível Superior - Brasil (CAPES) - Finance Code 001.

\section{References}

Aparicio, A. F., Vela, F. L. G., Sánchez, J. L. G., and Montes, J. L. I. (2012). Analysis and application of gamification. In Proceedings of the 13th International Conference on InteracciÓN Persona-Ordenador, INTERACCION '12, pages 17:1-17:2. ACM.

Banfield, J. and Wilkerson, B. (2014). Increasing student intrinsic motivation and selfefficacy through gamification pedagogy. Contemporary Issues in Education Research (Online), 7(4):291.

Brühlmann, F. (2013). Gamification From the Perspective of Self-Determination Theory and Flow. Bachelor thesis, University of Basel.

Conejo, G. G. (2018). Detalhando a motivação em um processo de gamificação. Bachelor thesis, Bacharelado em Ciência da Computação, Universidade do Estado de Santa Catarina, Joinville.

Deci, E. L. and Ryan, R. M. (2008). Self-determination theory: A macrotheory of human motivation, development, and health. Canadian psychology, 49(3):182.

Deterding, S., Sicart, M., Nacke, L., O'Hara, K., and Dixon, D. (2011). Gamification. Using Game-design Elements in Non-gaming Contexts. In CHI'11 Extended Abstracts on Human Factors in Computing Systems, CHI EA '11, pages 2425-2428.

Ewais, S. and Alluhaidan, A. (2015). Classification of stress management mhealth apps based on octalysis framework. In Twenty-first Americas Conference on Information Systems, Puerto Rico.

Hanus, M. D. and Fox, J. (2015). Assessing the effects of gamification in the classroom: A longitudinal study on intrinsic motivation, social comparison, satisfaction, effort, and academic performance. Computers Education, 80:152-161.

Hassenzahl, M. and Tractinsky, N. (2006). User experience - a research agenda. Behaviour \& Information Technology, 25(2):91-97.

Klock, A. C. T. (2017). Análise da Influência da Gamificação na Interação, na Comunicaçãoe no Desempenho dos Estudantes em um Sistema de Hipermídia Adaptativo Educacional. Master's dissertation, Universidade do Estado de Santa Catarina, Joinville, SC, Brazil. 
Klock, A. C. T., Gasparini, I., and Pimenta, M. S. (2016). 5w2h framework: A guide to design, develop and evaluate the user-centered gamification. In Proceedings of the 15th Brazilian Symposium on Human Factors in Computing Systems, pages 1-10.

Mekler, E. D., Brühlmann, F., Tuch, A. N., and Opwis, K. (2017). Towards understanding the effects of individual gamification elements on intrinsic motivation and performance. Computers in Human Behavior, 71:525 - 534.

Pink, D. H. (2011). Drive: The surprising truth about what motivates us. Penguin.

Ryan, R. M. and Deci, E. L. (2000). Intrinsic and extrinsic motivations: Classic definitions and new directions. Contemporary educational psychology, 25(1):54-67.

Ryan, R. M., Mims, V., and Koestner, R. (1983). Relation of reward contingency and interpersonal context to intrinsic motivation: A review and test using cognitive evaluation theory. Journal of personality and Social Psychology, 45(4):736.

Werbach, K. and Hunter, D. (2012). For the win: How game thinking can revolutionize your business. Wharton Digital Press, Philadelphia. 